\title{
A Migrating Laparoscopic Gastric Band Unveiling an Appendicular Cancer: A Rare Event
}

\section{Vishnoi $\mathbf{V}^{*}$ and Mehotra $\mathbf{R}$}

Department of Surgery, Hunter New England Health, Australia

\begin{abstract}
We present a 60-year-old male who was incidentally found to have an appendicular cancer. This resulted from a diagnostic laparoscopy, looking for a migrated laparoscopic gastric band. This case highlights the ongoing complications and issues associated with laparoscopic gastric bands, however the sequence of events leading to this patient's eventual diagnosis of appendicular cancer is very rare and worth discussing.
\end{abstract}

Keywords: Appendicular cancer; Laparoscopic gastric band

\section{Introduction}

Metabolic syndrome is on the increase in the Western World. Its complications are becoming apparent and a burden on the health sector. Obesity has multiple impacts on one's overall health, including cardiovascular (Ischaemic heart disease), endocrine (diabetes mellitus), and orthopaedic (advanced osteoarthritis) just to mention a few. In the last 20 years, surgical advances have attempted to counteract it with the development of obesity surgery [1]. Procedures such as gastric sleeves, duodenal switches and laparoscopic gastric bands have become quite popular [2]. These procedures are not without their complications. gastric bands. Gastric bands have been associated with a wide range of complications, which has made the procedure unfavourable amongst clinicians. In this case report, we present a 60 -year-old male who was found to incidentally have a pseudomyxoma peritonei, after a diagnostic laparoscopy revealed a displaced, migrated gastric band that had surrounded the appendix. This is an incredibly rare event that highlights the ongoing complications associated with laparoscopic gastric bands. However, the sequence of events leading to the patients' eventual diagnosis is exceptionally rare, and worth discussing.

\section{Case Report}

We present a 60-year-old male with a background history of obesity, type II diabetes mellitus and hypertension. He was a smoker and a social drinker. After consultation with a bariatric surgeon, the patient decided to undergo placement of a laparoscopic gastric band to treat his overall metabolic syndrome. The procedure was performed without any pre, intra or immediate post-operative complications and the patient was discharged two days following the procedure. The procedure was initially successful with the patient losing 15 kilograms, with a notable improvement in his blood pressure and blood sugar level. His recovery, however, was complicated by the development of vague periumbilical abdominal pain one-year post operatively. The pain was intermittent and colicky. There was no nausea, vomiting, change in bowel habits or fevers. Serial abdominal examinations by general practitioner and the specialist in charge demonstrated a soft and nontender abdomen. The patient was managed with simple analgesia and reassurance, however he continued to suffer from abdominal pain. After frequent visits to the GP, a computed tomography scan of the abdomen and pelvis was organized. The CT scan demonstrated a radioopaque circular structure in the right iliac fossa (Figures 1 and 2). Its appearance consistent with a migrated gastric band surrounding a blind luminal structure (potentially the appendix) with associated stranding. The patient was admitted into hospital for further investigations. His white cell count and $\mathrm{C}$ - reactive protein was 8 and 35 respectively. The

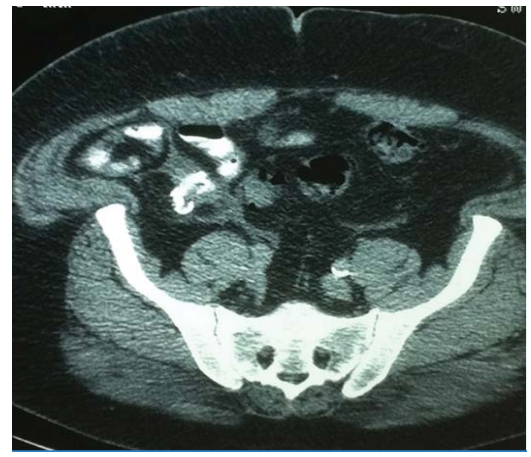

Figure 1: An axial view of the CT abdomen/pelvis, showing a radio-opaque structure in the right iliac fossa.

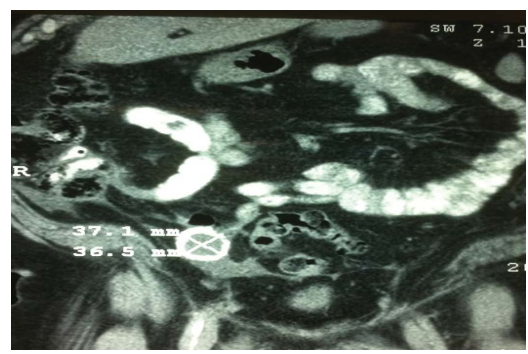

Figure 2: A coronal view of the CT abdomen/pelvis showing a radio-opaque structure in the right iliac fossa.

patient proceeded to have a diagnostic laparoscopy. The laparoscopy confirmed the radiological findings. The gastric band had migrated into the right iliac fossa, surrounded and trapped the tip of appendix, with gelatinous fluid in the pelvis. The appendix macroscopically appeared enlarged, erythematous, and consistent with appendicitis without perforation (Figure 3). A laparoscopic appendectomy was performed without complication and both the appendix and gastric band were

*Corresponding author: Vishnoi V, Department of Surgery, Hunter New England Health, Australia, Tel: +61411676282; E-mail: Veral.vishnoi@hnehealth.nsw.gov.au

Received August 10, 2018; Accepted August 17, 2018; Published August 21, 2018

Citation: Vishnoi V, Mehotra R (2018) A Migrating Laparoscopic Gastric Band Unveiling an Appendicular Cancer: A Rare Event. J Clin Case Rep 8: 1157. doi: 10.4172/2165-7920.10001157

Copyright: (c) 2018 Vishnoi V, et al. This is an open-access article distributed under the terms of the Creative Commons Attribution License, which permits unrestricted use, distribution, and reproduction in any medium, provided the original author and source are credited. 


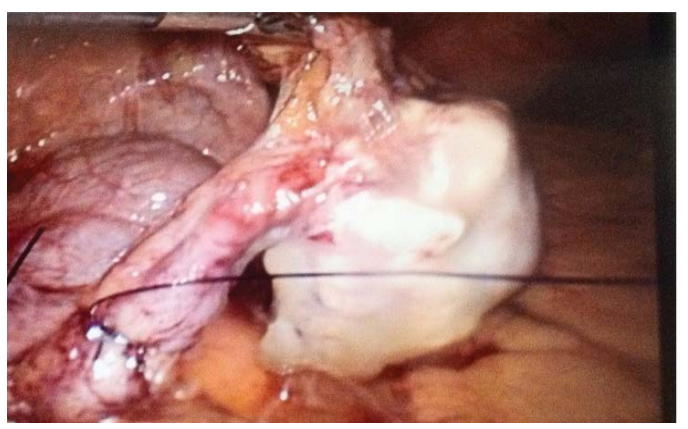

Figure 3: A laparoscopic view of the gastric band surrounding part of the appendix.

removed via endobag. The patient had an uncomplicated recovery and was discharged home day 1 post operatively. The histopathology returned as mucinous cystadenocarcinoma with a high possibility of pseudomyxoma peritonei (PMP), a rare and very unexpected finding. The patient was referred to a tertiary center for ongoing management.

\section{Discussion}

Laparoscopic adjustable gastric banding (LAGB) was introduced in the mid-1980s and gained popularity amongst surgeons as treatment of morbid obesity because it is minimally invasive and theoretically reversible [3]. However, high complication rates have led to a marked decline in LAGB insertion from 42.3\% to $17.8 \%$ (between 2008 to 2011) in Australia [4]. Its complications include, gastric perforation, band displacement and pouch enlargement [5]. There have been reports in the literature where gastric band migration has occurred following erosion into the stomach lumen, travelling distally into the small bowel [6] and further [7]. Furthermore, there have been instances of gastric band displacement, with associated small bowel perforation and small bowel obstruction [4]. These complications range from acute, to sub- acute in presentation. Furthermore, laparoscopic gastric bands have been shown to achieve poor results in terms of patient satisfaction in the long term, due the nature of the complications [8].

\section{Conclusion}

We present a rare complication of LAGB, resulting in early diagnosis of a serious pathology. It is possible that this patient may have remained asymptomatic, with further disease progression of his PMP if the LAGB had not found its way down to the appendix. This case highlights the ongoing complications of LAGB, however in this instance it is perhaps to the patients benefit.

\section{References}

1. Arterburn DE, Courcoulas AP (2014) Bariatric surgery for obesity and metabolic conditions in adults. The BMJ 349: 3961.

2. Karmali S, Stoklossa C, Sharma A, Stadnyk J, Christiansen S, et al. (2010) Bariatric surgery: Clinical review. Can Fam Physician p: 56

3. LK (1986) Silicone gastric banding: A simple and effective operation for morbid obesity. Contemp Surg p: 28

4. Abeysekera ALJ, Ghosh S, Hacking C (2017) Migration of eroded laparoscopic adjustable gastric band causing small bowel obstruction and perforation. BMJ Case Rep 2: 1 .

5. Eid IBD, Sharma A, Sherman V, Karmali S (2009) Complications associated with adjustable gastric banding for morbid obesity: A surgeon's guide. Can $J$ Surg p: 54.

6. Collado-Pachecom DRTL, Arias-Rivera M, Ortega-Carbonel A, OlivaresValles A, Alonsno-Prada A, et al. (2016) Endoscopic extraction of adjustable gastric bands after intragastric migration as a complication of bariatric surgery: Technique and advice. Endoscopy international open 4: 673-677.

7. Bassam A (2012) Unusual gastric band migration outcome: Distal small bowe obstruction and coming out per-rectum. Pan Africa Medical Journal 13: 59

8. Kowalewski PK, Olszewski R, Kwiatkowski A, Gałązka-Świderek N, Cichoń $\mathrm{K}$, et al. (2017) Life with a gastric band. Long-term outcomes of laparoscopic adjustable gastric banding: A retrospective study. Obesity Surgery 27: 12501253. 